\title{
Artvin Borçka Karagöl Tabiat Parkı'nın Ornitofaunası
}

\author{
Mehmet Mustafa ALAR, Hümeyra NERGIZ** \\ Bitlis Eren Üniversitesi, Fen-Edebiyat Fakültesi, Biyoloji Bölümü, Bitlis \\ (ORCID: 0000-0001-7817-034X) (ORCID: 0000-0001-5588-6134)
}

\begin{abstract}
$\ddot{\mathbf{O z}}$
Bu çalışmada Artvin Borçka Karagöl Tabiat Parkı'nda bulunan kuş türleri araştırılmıştır. Araştırmada Mart 2017 Aralık 2018 tarihleri arasında gerçekleştirilen gözlem sonuçları değerlendirilmiş, 14 takım ve 36 familyaya ait 88 kuş türü tespit edilmiştir. Bu türlerden 34'ü yerli (\% 38.6), 32'si yaz ziyaretçisi (\% 36.3), 3'ü kış ziyaretçisi (\% 3.4) ve 19'u transit göçerdir (\% 21.7). Passeriformes takımı alanda en fazla türle (47 tür) temsil edilirken, Podicipediformes, Gruiformes, Cuculiformes ve Bucerotiformes takımlarına ait alanda birer tür tespit edilmiştir. Alandaki kuşların IUCN (Uluslararası Doğa Koruma Birliği) kriterlerine göre tehlike durumları; 1 tür EN (Falco cherrug), 2 tür NT (Milvus milvus, Falco vespertinus), 1 tür VU (Clanga clanga) olarak verilmiştir. Ayrıca Bern Sözleşmesine göre alandaki kuş türlerinin 51 tanesi Ek II listesinde, 27 tanesi ise EK III listesinde yer almaktadır.
\end{abstract}

Anahtar kelimeler: Kuşlar, Sulak Alan, Biyoçeşitlilik, Ornitofauna.

\section{Ornithofauna of Artvin Borçka Karagöl Nature Park}

\begin{abstract}
In this study, bird species at Artvin Borçka Karagöl Nature Park were researched. In this research, conducted between years of March 2017-December 2018, 88 bird species belonging to 36 families of 14 ordo were identified. Of these species, 34 (\% 38.6) were resident, 32 (\% 36.3) were summer migrant, 3 (\% 3.4) were winter migrant and 19 (\% 21.5) were transit migrant. These species were belonged to different IUCN (International Union for Conservation of Nature) Red List Categories including, Falco cherrug-EN, Milvus milvus and Falco vespertinusNT and Clanga clanga-VU. According to Bern Convention Red List Categories, all of observed species were categorized as; 51 in Appendix II and while 27 in Appendix III.
\end{abstract}

Keywords: Birds, Wetland, Biodiversity, Ornithofauna.

\section{Giriş}

Sulak alanlar sahip oldukları biyolojik çeşitlilik nedeniyle yeryüzünün en önemli ekosistemlerindendir. Farklı ekosistemlerin bir arada bulunması ve habitat çeşitliliğinin fazla olması bu alanlardaki tür çeşitliliğini arttırmaktadır. Her ekosistemin iklimi, biyolojik ve topografik özellikleri birbirinden farklıdır. Bu durum ekosistem çeşitliliğine neden olur. Ekosistemlerin orman, göl, çöl, dağ, sazlık, akarsu, okyanus gibi çeşitleri vardır. Bu çeşitlilik arttıkça, ekosistemde yer alan habitat ve tür çeşitliliği de artar. Ancak biyoçeşitliliğin bileşenleri olarak ekosistemler ve türler hızla bozulma ya da yok olma tehdidi altındadır $[1,2]$.

Türkiye, konumu, topografik yapısı ve buna bağlı olarak ortaya çıkan iklim koşulları nedeniyle biyoçeşitlilik açısından son derece zengindir. Flora ve faunasındaki bu zenginliğin yanı sıra, endemizm olgusu Anadolu'nun doğal yapısını daha da çekici hale getirmektedir. Bu doğal yapı çeşitliliği Anadolu'ya kıta özelliği vermektedir [3]. Ülkemiz birç̧ok bakımdan olduğu gibi, kuş türü varlığ1 bakımından da kıta özelliği göstermektedir. Batı Palearktik bölgeyi güneydeki kışlama alanlarına bağlayan Anadolu aynı zamanda zengin bir kuş faunasına sahiptir. Palearktik bölgedeki dört kuş göç yolundan ikisi Anadolu üzerinden geçmektedir. Bunlardan ilki; Trakya'dan ülkemize girip Boğaziçi

"Sorumlu yazar: h.nergiz@beu.edu.tr

Geliş Tarihi: 22.06.2019, Kabul Tarihi: 23.07.2019 
üzerinden Anadolu'ya geçen Kuzeybatı-Güney göç rotasıdır. Kuzeydoğu-Güney göç rotası ise Doğu Karadeniz'den ülkemize girip, Çoruh Nehri üzerinden geçerek Doğu Anadolu'ya doğru yayılmaktadır [4].

$\mathrm{Bu}$ çalışmada sahip olduğu floristik ve faunistik zenginliği ve peyzaj özellikleri sebebiyle yüksek ekoturizm potansiyeline sahip olan Artvin Borçka Karagöl Tabiat Parkı'nda konaklayan ve üreyen kuş türleri ile türlere ve yaşam alanlarına yönelik tehdit unsurları ve alınması gereken tedbirler araştırılmıştır.

\section{Materyal ve Metot}

\section{1. Çalışma alanının tanıtımı}

Karagöl Tabiat Parkı, Artvin ili Borçka ilçesi sınırları içerisinde, Karçal Dağları'nın kuzeybatısında, deniz seviyesinden 1450-1480 m aralığında yer almaktadır (Şekil 1).

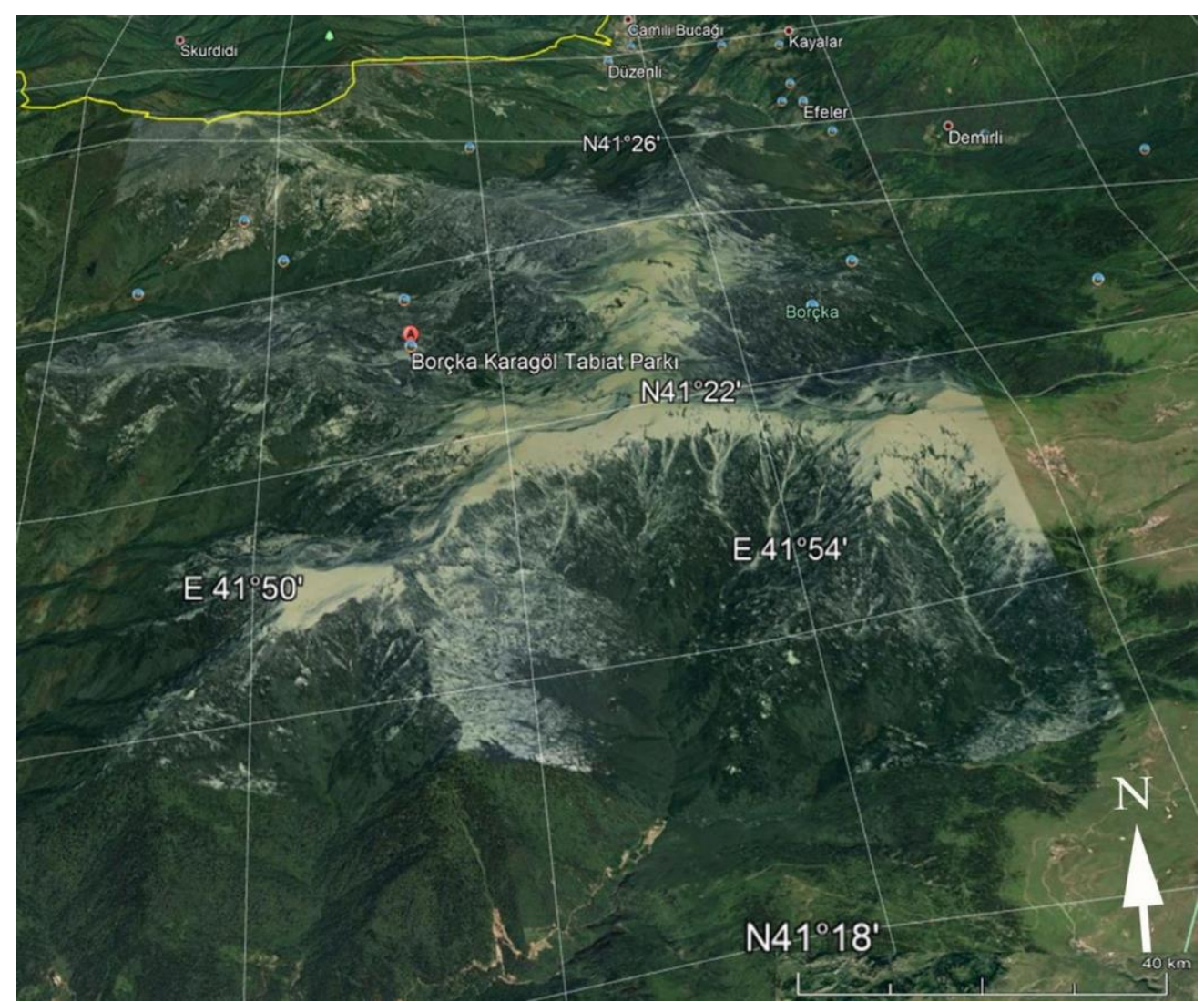

Şekil 1. Araştırma alanının uydu görüntüsü (Google earth image-2018 GeoEye).

Karagöl, maksimum derinliği 7 m olan 10 ha büyüklügünde bir tatlı su gölüdür. Akarsular ve yağışlarla beslenir. Savgule ve Heba dereleri Karagöl'ü besleyen en önemli kaynaklardır [1, 5, 7]. Karagöl ve çevresindeki 368 ha'lık alan; fauna ve flora zenginliği, jeomorfolojik, jeolojik ve peyzaj özellikleri sebebiyle 14.08.2002 tarihinde Doğa Koruma ve Milli Parklar Genel Müdürlüğü tarafindan "Tabiat Parkı" kapsamına alınmıştır. Karadeniz ikliminin hüküm sürdüğü Artvin Borçka Karagöl Tabiat Park1 ve çevresinde kışlar 1lık, yazlar sıcak ve bol yağışlıdır. Yı1lık ortalama yağış $1268.2 \mathrm{~mm}$, ortalama sıcaklık ise $13{ }^{\circ} \mathrm{C}^{\prime}$ dir $[7,8]$. Nemli oluşundan dolayı doğal vejetasyonun çeşitlilik gösterdiği sahada endemizm oranı \%1.1'dir. Ekolojik olarak bitkilerin varlığını sürdürmelerine uygun olan göl çevresinde özellikle Ladin (Picea orientalis), Sarıçam (Pinus sylvestris), Doğu Karadeniz Göknarı (Abies nordmanniana), Doğu Kayını (Fagus orientalis) ve Ceviz (Juglans regia) türlerinin hakim olduğu karışık ormanlık alan bulunmaktadır $[9,10]$. 


\subsection{Metot}

Artvin-Borçka Karagöl Tabiat Parkı'nda Mart 2017-Aralık 2018 tarihleri arasında gerçekleştirilen arazi çalışmaları aylık periyotlarla çalışma takvimine bağlı olarak yürütülmüştür. Mevsimsel olarak gün uzunluğuna bağlı olarak ayarlanan gözlemler genellikle kuş türlerinin aktif olduğu $6^{00}-19^{00}$ saatleri arasında yapılmıştır. Çalışma sahasında varlığını sürdüren kuş türlerinin, populasyonları, çalışma sahasına geliş ve ayrılış zamanları, alandaki yayılışları, statüleri ve kırmızı listedeki yerleri tespit edilmiştir.

Çalışma alanındaki kuş türleri Dobinson'un (1976) "Kareler (Raster Kartlama)" yöntemi kullanılarak tespit edilmiştir [11]. Populasyon büyüklügünü belirlemek için yapılan sayımlar genellikle çalışma sahasına hâkim olan noktalardan belli bir hat dikkate alınarak teleskop, dürbün ve çıplak göz yardımı ile bütün alanın taranması şeklinde yapılmıştır. Ayrıca gözlemler sırasında türlerin morfolojik karakterleri, sesleri, uçuş özellikleri, habitat seçimleri ve yuvalanma şekilleri gibi özelliklerinden de faydalanılmıştır. Kuluçka ve yuva yerlerini gözlemek amacı ile arazide yine bir hat boyu belli bir genişlik dikkate alınıp yürünerek gözlemler gerçekleştirilmiştir. Çalışmalarda; alanın 1/25000'lik haritası, arazi gözlem kartları, dürbün (Kodak 8 x 40), teleskop (Konuspot 100 mm), numaratör, fotoğraf makinesi (Canon Eos 7D, 400mm objektif), bataklık giysileri ve teşhis kitapları [12-15] kullanılmıştır.

\section{Bulgular ve Tartışma}

Artvin-Borçka Karagöl Tabiat Parkı'nda Mart 2017-Aralık 2018 tarihleri arasında gerçekleştirilen gözlem ve çalışmalar sonucunda alanda toplam 14 takım ve 36 familyaya ait 88 kuş türü tespit edilmiştir. Bu türlerin alandaki göç durumları dikkate alındığında; 34'ü yerli (\% 38.6), 32'si yaz ziyaretçisi (\% 36.3), 3'ü kış ziyaretçisi (\% 3.4) ve 19'u transit göçerdir (\% 21.7) (Tablo 1).

Tablo1. Artvin Borçka Karagöl Tabiat Parkı'nda tespit edilen kuş türleri ve statüleri

\begin{tabular}{|c|c|c|c|c|c|c|}
\hline Ordo & Familya & Tür & $\begin{array}{l}\text { Göç } \\
\text { Statüsü }\end{array}$ & IUCN & Bern & CITES \\
\hline PODICIPEDIFORMES & PODICIPEDIDAE & Tachybaptus ruficollis & $\overline{\mathrm{Y}}$ & $\overline{\mathrm{LC}}$ & EKII & - \\
\hline \multirow[t]{3}{*}{ PELECANIFORMES } & ARDEIDAE & Ardea alba & YZ & $\mathrm{LC}$ & EKII & - \\
\hline & & Ardea cinerea & YZ & $\mathrm{LC}$ & EKIII & - \\
\hline & & Egretta garzetta & YZ & $\mathrm{LC}$ & EKII & - \\
\hline \multirow[t]{11}{*}{ ACCIPITRIFORMES } & ACCIPITRIDAE & Pernis apivorus & TG & $\mathrm{LC}$ & EKII & EKII \\
\hline & & Milvus milvus & TG & NT & EKII & EKII \\
\hline & & Milvus migrans & $\mathrm{Y}$ & $\mathrm{LC}$ & EKII & EKII \\
\hline & & Clanga pomarina & TG & $\mathrm{LC}$ & EKII & EKII \\
\hline & & Clanga clanga & TG & VU & EKII & EKII \\
\hline & & Accipiter gentilis & $\mathrm{YZ}$ & $\mathrm{LC}$ & EKII & EKII \\
\hline & & Accipitter nisus & $\mathrm{Y}$ & $\mathrm{LC}$ & EKII & EKII \\
\hline & & Buteo buteo & Y & $\mathrm{LC}$ & EKII & EKII \\
\hline & & Buteo rufinus & $\mathrm{Y}$ & $\mathrm{LC}$ & EKII & EKII \\
\hline & & Hieraaetus pennatus & TG & $\mathrm{LC}$ & EKII & EKII \\
\hline & & Aquila chrysaetos & TG & $\mathrm{LC}$ & EKII & EKII \\
\hline \multirow[t]{6}{*}{ FALCONIFORMES } & FALCONIDAE & Falco tinnunculus & $\mathrm{Y}$ & $\mathrm{LC}$ & EKII & EKII \\
\hline & & Falco naumanni & TG & LC & EKII & EKII \\
\hline & & Falco subbuteo & YZ & $\mathrm{LC}$ & EKII & EKII \\
\hline & & Falco vespertinus & TG & NT & EKII & EKII \\
\hline & & Falco columbarius & $\mathrm{KZ}$ & $\mathrm{LC}$ & EKII & EKII \\
\hline & & Falco cherrug & TG & EN & EKII & EKII \\
\hline GRUIFORMES & RALLIDAE & Fulica atra & $\mathrm{Y}$ & LC & EKIII & - \\
\hline \multirow[t]{5}{*}{ CHARADRIIFORMES } & CHARADRIIDAE & Charadrius dubius & YZ & $\mathrm{LC}$ & EKII & - \\
\hline & SCOLOPACIDAE & Scolopax rusticola & TG & $\mathrm{LC}$ & EKIII & - \\
\hline & & Tringa ochropus & TG & LC & EKII & - \\
\hline & & Tringa glareola & YZ & LC & EKII & - \\
\hline & LARIDAE & Larus ridibundus & $\mathrm{Y}$ & $\mathrm{LC}$ & EKIII & - \\
\hline \multirow{2}{*}{ COLUMBIFORMES } & COLUMBIDAE & Columba livia & $\mathrm{Y}$ & $\mathrm{LC}$ & EKIII & . \\
\hline & & Columba palumbus & TG & $\mathrm{LC}$ & & - \\
\hline CUCULIFORMES & CUCULIDAE & Cuculus canorus & $\mathrm{YZ}$ & LC & EKIII & - \\
\hline
\end{tabular}




\begin{tabular}{|c|c|c|c|c|c|c|}
\hline Ordo & Familya & Tür & $\begin{array}{l}\text { Göç } \\
\text { Statüsü } \\
\end{array}$ & IUCN & Bern & CITES \\
\hline \multirow[t]{3}{*}{ STRIGIFORMES } & STRIGIDAE & Athene noctua & $\bar{Y}$ & $\overline{\mathrm{LC}}$ & EKII & EKII \\
\hline & & Bubo bubo & Y & $\mathrm{LC}$ & EKII & EKII \\
\hline & & Strix aluco & $\mathrm{Y}$ & $\mathrm{LC}$ & EKII & EKII \\
\hline \multirow[t]{3}{*}{ CAPRIMULGIFORMES } & CAPRIMULGIDAE & $\begin{array}{l}\text { Caprimulgus } \\
\text { europaeus }\end{array}$ & $\mathrm{YZ}$ & $\mathrm{LC}$ & EKII & - \\
\hline & APODIDAE & Apus apus & TG & $\mathrm{LC}$ & EKIII & - \\
\hline & & Tachymarptis melba & TG & $\mathrm{LC}$ & EKII & - \\
\hline \multirow[t]{2}{*}{ CORACIIFORMES } & MEROPIDAE & Merops apiaster & $\mathrm{YZ}$ & $\mathrm{LC}$ & EKII & - \\
\hline & CORACIIDAE & Coracias garrulus & $\mathrm{YZ}$ & $\mathrm{LC}$ & EKII & - \\
\hline BUCEROTIFORMES & UPUPIDAE & Uрира ерорs & $\mathrm{YZ}$ & $\mathrm{LC}$ & EKIII & - \\
\hline \multirow[t]{2}{*}{ PICIFORMES } & PICIDAE & Dendrocopos major & $\mathrm{Y}$ & $\mathrm{LC}$ & EKII & - \\
\hline & & Dendrocopos syriacus & $\mathrm{Y}$ & $\mathrm{LC}$ & EKII & - \\
\hline \multirow{47}{*}{ PASSERIFORMES } & ALAUDIDAE & Alauda arvensis & $\mathrm{Y}$ & $\mathrm{LC}$ & EKIII & - \\
\hline & & Lullula arborea & Y & $\mathrm{LC}$ & EKIII & - \\
\hline & CINCLIDAE & Cinclus cinclus & $\mathrm{Y}$ & - & - & - \\
\hline & TROGLODYTIDAE & $\begin{array}{l}\text { Troglodytes } \\
\text { troglodytes }\end{array}$ & $\mathrm{Y}$ & $\mathrm{LC}$ & EKII & - \\
\hline & MOTACILLIDAE & Motacilla flava & YZ & $\mathrm{LC}$ & EKII & - \\
\hline & & Motacilla citreola & YZ & $\mathrm{LC}$ & EKIII & - \\
\hline & & Motacilla cinerea & YZ & $\mathrm{LC}$ & EKII & - \\
\hline & & Motacilla alba & YZ & $\mathrm{LC}$ & EKIII & - \\
\hline & SITTIDAE & Sitta europaea & $\mathrm{Y}$ & $\mathrm{LC}$ & EKIII & - \\
\hline & CERTHIIDAE & Certhia familiaris & $\mathrm{Y}$ & $\mathrm{LC}$ & EKIII & - \\
\hline & MUSCICAPIDAE & Muscicapa striata & YZ & $\mathrm{LC}$ & EKII & - \\
\hline & & Ficedula parva & $\mathrm{YZ}$ & $\mathrm{LC}$ & EKII & - \\
\hline & & Erithacus rubecula & $\mathrm{Y}$ & $\mathrm{LC}$ & EKII & - \\
\hline & & $\begin{array}{l}\text { Luscinia } \\
\text { megarhynchos }\end{array}$ & $\mathrm{YZ}$ & $\mathrm{LC}$ & EKII & - \\
\hline & & $\begin{array}{l}\text { Phoenicurus } \\
\text { phoenicurus }\end{array}$ & $\mathrm{YZ}$ & $\mathrm{LC}$ & EKII & - \\
\hline & & Saxicola torquata & $\mathrm{YZ}$ & $\mathrm{LC}$ & - & - \\
\hline & TURDIDAE & Turdus torquatus & $\mathrm{YZ}$ & $\mathrm{LC}$ & EKIII & - \\
\hline & & Turdus merula & $\mathrm{Y}$ & $\mathrm{LC}$ & EKIII & - \\
\hline & & Turdus philomelos & $\mathrm{KZ}$ & $\mathrm{LC}$ & EKIII & - \\
\hline & SYLVIIDAE & Sylvia curruca & $\mathrm{YZ}$ & $\mathrm{LC}$ & EKII & - \\
\hline & & Sylvia communis & $\mathrm{YZ}$ & $\mathrm{LC}$ & EKII & - \\
\hline & & Sylvia borin & $\mathrm{YZ}$ & $\mathrm{LC}$ & EKII & - \\
\hline & & Sylvia nisoria & TG & $\mathrm{LC}$ & EKII & - \\
\hline & REGULIDAE & Regulus regulus & $\mathrm{Y}$ & $\mathrm{LC}$ & EKII & - \\
\hline & PHYLLOSCOPIDAE & Phylloscopus collybita & TG & $\mathrm{LC}$ & EKIII & - \\
\hline & & $\begin{array}{l}\text { Phylloscopus } \\
\text { sindianus }\end{array}$ & TG & $\mathrm{LC}$ & EKIII & - \\
\hline & PARIDAE & Parus major & $\mathrm{Y}$ & $\mathrm{LC}$ & EKII & - \\
\hline & & Periparus ater & $\mathrm{Y}$ & $\mathrm{LC}$ & EKII & - \\
\hline & & Cyanistes caeruleus & $\mathrm{Y}$ & $\mathrm{LC}$ & EKII & - \\
\hline & AEGITHALIDAE & Aegithalos caudatus & $\mathrm{Y}$ & $\mathrm{LC}$ & EKIII & - \\
\hline & LANIIDAE & Lanius collurio & YZ & $\mathrm{LC}$ & EKII & - \\
\hline & & Lanius senator & TG & $\mathrm{LC}$ & EKII & - \\
\hline & & Lanius nubicus & TG & $\mathrm{LC}$ & EKII & - \\
\hline & CORVIDAE & Corvus corone & $\mathrm{Y}$ & $\mathrm{LC}$ & - & - \\
\hline & & Corvus corax & $\mathrm{Y}$ & $\mathrm{LC}$ & EKIII & - \\
\hline & & Garrulus glandarius & $\mathrm{Y}$ & $\mathrm{LC}$ & - & - \\
\hline & & Pica pica & Y & $\mathrm{LC}$ & - & - \\
\hline & STURNIDAE & Sturnus vulgaris & $\mathrm{Y}$ & $\mathrm{LC}$ & - & - \\
\hline & PASSERIDAE & Passer montanus & $\mathrm{Y}$ & $\mathrm{LC}$ & EKIII & - \\
\hline & & Passer domesticus & $\mathrm{Y}$ & $\mathrm{LC}$ & - & - \\
\hline & FRINGILLIDAE & Fringilla coelebs & $\mathrm{YZ}$ & $\mathrm{LC}$ & EKIII & - \\
\hline & & $\begin{array}{l}\text { Fringilla } \\
\text { montifringilla }\end{array}$ & $\mathrm{KZ}$ & $\mathrm{LC}$ & EKIII & - \\
\hline & & Serinus serinus & $\mathrm{YZ}$ & $\mathrm{LC}$ & EKIII & - \\
\hline & & Chloris chloris & $\mathrm{YZ}$ & $\mathrm{LC}$ & - & - \\
\hline & & Carduelis carduelis & $\mathrm{YZ}$ & $\mathrm{LC}$ & EKIII & - \\
\hline & EMBERIZIDAE & Emberiza calandra & $\mathrm{YZ}$ & $\mathrm{LC}$ & EKIII & - \\
\hline & & $\begin{array}{l}\text { Emberiza } \\
\text { melanocephala }\end{array}$ & $\mathrm{YZ}$ & $\mathrm{LC}$ & EKIII & - \\
\hline
\end{tabular}


Türlerin takımlara göre dağılımları; Podicipediformes 1, Pelecaniformes 3, Accipitriformes 11, Falconiformes 6, Gruiformes 1, Charadriiformes 5, Columbiformes 2, Cuculiformes 1, Strigiformes 3, Caprimulgiformes 3, Coraciiformes 2, Bucerotiformes 1, Piciformes 2 ve Passeriformes 47 türle temsil edilmektedir. Artvin Borçka Karagöl Tabiat Parkı'nda Passeriformes takımı en fazla, Podicipediformes, Gruiformes, Cuculiformes ve Bucerotiformes takımları ise en az türle temsil edilmektedir (Şekil 2).

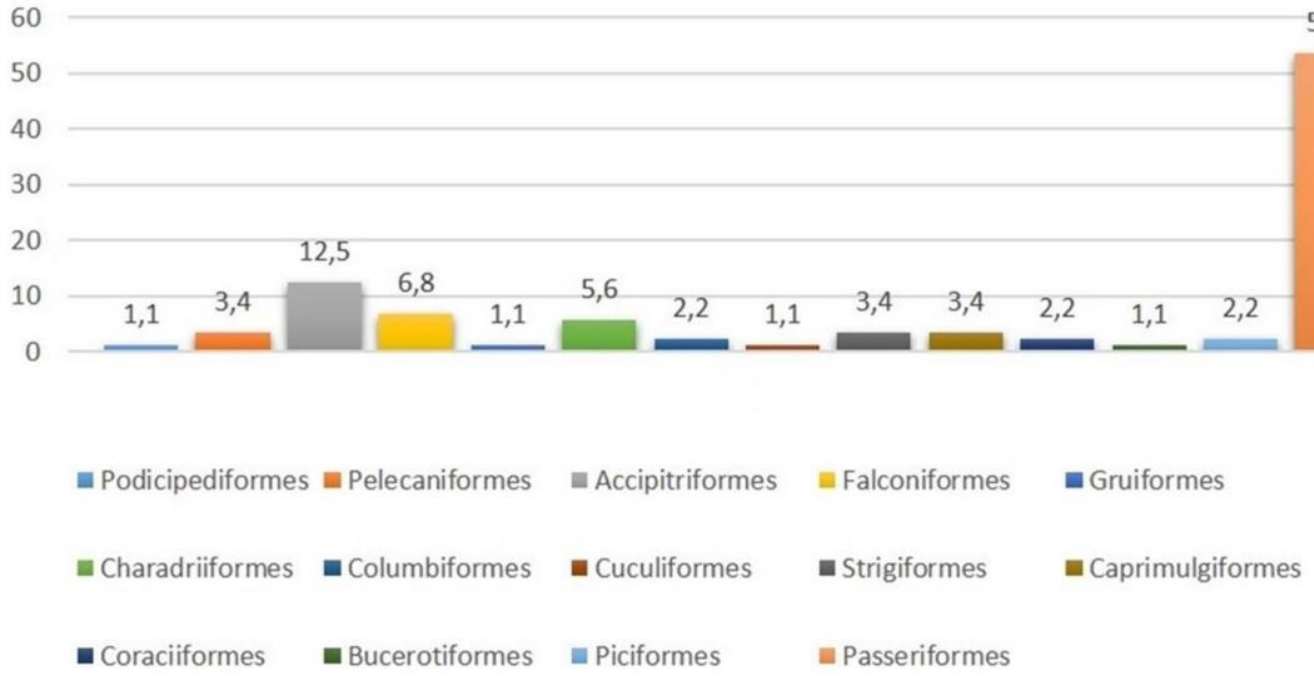

Şekil 2. Alandaki kuş türlerinin takımlara göre yüzdelik oranları

Alanda gözlemlenen kuş türlerinin IUCN tehlike kategorileri dikkate alındığında; 1 tür EN "Tehlike altında" (Falco cherrug), 2 tür NT "Tehdide aç1k" (Milvus milvus ve Falco vespertinus) ve 1 tür de VU "Zarar görebilir=Duyarlı" (Clanga clanga) kategorisinde yer almaktadır. Geriye kalan 84 tür ise LC "Asgari endişe=Düşük riskli" kategorisinde bulunmaktadır. Alandaki türlerin Bern Sözleşmesi kapsamında değerlendirilmesi yapıldığında 51 tür EK-II'de; yani "Mutlak Koruma Altındaki Türler" arasında yer almaktadır. Bunlar özellikle Accipitridae, Muscicapidae, ve Motacillidae familyalarına mensup türlerdir. Ayrıca, Bern sözleşmesinin EK-II no'lu listesinde yer alan türlerden 17 tanesi Y statüsünde yer alırken 19 tanesi YZ statüsündedir. Alandaki 27 kuş türü ise Bern sözleşmesinin EK III listesinde, CITES sözleşmesine göre de 20 tür EK-II listesindedir.

Alandaki 88 kuş türünün yayılıș alanı ve göç statüleri; Kiziroğlu (2008), Green Moorhouse (1995) ve Kirwan vd. (1998)'in verileriyle uygunluk göstermektedir [16, 17]. Kahraman vd. (2016)'ya göre Büyük ak balıkçıl (Ardea alba) Doğu Karadeniz için kış ziyaretçisi, Gri balıkçıl (Ardea cinerea) ise yerli tür olarak verilmiştir [18]. Çalışmamızda ise her iki türün de Karagöl'de yaz ziyaretçisi olduğu tespit edilmiştir. Aynı çalışmada bölge için kış ziyaretçisi olarak tanımlanan Çulluk (Scolopax rusticola) ve Yeşil düdükçün (Tringa ochropus) çalışmamız süresince sadece birkaç kez sonbahar döneminde gözlenmiştir.

Göktürk vd. (2008)'nin Artvin için yaz ziyaretçisi olarak belirledikleri Arı şahini (Pernis apivorus), Kızıl çaylak (Milvus milvus), Ala doğan (Falco vespertinus) ve Ulu doğan (Falco cherrug) sadece göç döneminde transit göçer tür olarak gözlenmiştir [19].

\section{Sonuç ve Öneriler}

Artvin Borçka Karagöl Tabiat Parkı iklimi, topografik özellikleri ve konumu itibariyle birçok kuş türünün konaklaması ve üremesi için oldukça uygun bir alandır. Tabiat Parkı'nın sınırları içindeki ormanlık alanın özellikle ötücüler ve göç dönemindeki yırtıcılar tarafından tercih edildiği belirlenmiştir. Ancak yaz aylarında artış gösteren alandaki rekreasyonel faaliyetlerin özellikle alanda kuluçkaya yatan ötücüleri ve gölde konaklayan su kuşlarını büyük ölçüde etkilediği görülmüştür. Alanının yakınındaki çayırlık alanlarda yaz aylarında aşırı bir hayvan otlatması söz konusu olmaktadır. Bu durum bu alanlarda doğal dengenin bozulmasına, orman tahribatı ve yüksek eğim toprak erozyonuna sebep olmaktadır. Bütün bu olumsuzluklara rağmen alanda konaklayan yerli tür sayısının oldukça fazla olması alanın 
habitat zenginliğinin bir göstergesidir. Kızıl çaylak, Ala doğan, Ulu doğan ve Büyük orman kartalı gibi nesli dünya çapında tehlike altında olan türlerin göç döneminde bu alanda konaklıyor olması alanın önemini arttırmaktadır. Bütün bu veriler alanın tabiat parkı statüsünün devamlılığının sağlanmasının gerekliliğini vurgulamaktadır. Büyük bir turizm potansiyeli olan alanın sahip olduğu doğal zenginliğin sürdürülmesi için bu zenginliği oluşturan canlılardan biri olan kuşların korunması vazgeçilmez bir gerekliliktir. Bu değerlerin kazandırdığı potansiyeli, özellikle yöre halkı için ekonomik bir gelir kaynağına dönüştürme ekoturizm ile mümkün olacaktır. Bunun için yöre halkının, ekoturizm konusunda bilinçlendirilmesi ve eğitilmesi gerekmektedir. Tabiat Parkı'nı ziyaret eden insanların özellikle alandaki kuşların önemi konusundaki farkındalığını arttırmak için belli noktalara bilgilendirme ve ikaz tabelaları konulması faydalı olacaktır.

\section{Kaynaklar}

[1] Demirsoy A. 1999. Genel ve Türkiye Zoocoğrafyası "Hayvan Coğrafyası". Meteksan Yayınları, Ankara.

[2] Ketenoğlu O., Kurt L., Yiğit N., Akman Y. 2012. Ekolojik Sentez. Palme Yayınları, Ankara.

[3] Kiziroğlu İ. 2001. Ekolojik Potpuri. Tekav Yayınları, Ankara.

[4] Yiğit N., Saygı1ı F., Çolak E., Sözen M., Karataş A. 2008. Ornitoloji Ders Notları. Ümit Ofset Matbaası Yayınları, Ankara.

[5] Kopar İ., Sever R. 2008. Karagöl, (Borçka-Artvin). Ankara Üniversitesi Sosyal Bilimler Enstitüsü Dergisi, 11 (1): 21-38.

[6] Kolaylı S., Şahin B. 2009. Benthic Algae (Except Bacillariophyta) and Their Seasonal Variations in Karagöl Lake (Borçka, Artvin-Turkey).Turkish Journal of Botany, 33: 27-32.

[7] Anonim 2013. Artvin İlinde Doğa Turizmi Master Planı. Orman ve Su İşleri Bakanlığı Raporu, 12. Bölge Müdürlüğü, Artvin.

[8] Koçman A. 1993. Türkiye İklimi. Ege Üniversitesi Edebiyat Fakültesi Yayınları, İzmir.

[9] Özhatay N., Byfield A., Atay S. 2005. Türkiye'nin 122 Önemli Bitki Alanı. WWF Turkey, İstanbul.

[10] Eminağaoğlu Ö., Yüksek T., Gümüş S., Kurdoğlu O., Eraydın S. 2007. Borçka-Karagöl Tabiat Park1 ve Çevresinin Flora ve Vejetasyonu. Proje raporu, TOGTAG-3210. 190s.

[11] Dobinson H.M. 1976. Bird Count, A Practical Guide to Bird Survey. Penguin Books Ltd.Hormondsworth, England.

[12] Heinzel H., Fitter R., Parsiov J. 1995. Türkiye ve Avrupa'nın Kuşları. Doğal Hayatı Koruma Derneği Yayınevi, İstanbul.

[13] Harrison C., Greensmith A. 2000. Birds of the World. A. Dorling Kindersley Book, London.

[14] Kiziroğlu İ. 2008. Türkiye Kuşları Kırmızı Listesi. Desen Matbaası, Ankara.

[15] Svensson L., Mullarney K., Grant P.J., Zetterstrom D. 2011. Collins Bird Guide The Most Complete Guide to The Birds of Britain and Europe. Harper Collins.United Kingdom.

[16] Green I., Moorhouse N. 1995. A Birdwatchers' Guide to Turkey. Bird Watcher's Giides Prion Ltd., England.

[17] Kirwan G.M., Martins R.P., Eken G., Davidson P. 1998. Checklist of the Birds of Turkey. OSME Sandgrouse Supplement, USA.

[18] Kahraman H, Şeremet T, Hatinoğlu B, 2016. Doğu Karadeniz Kuşları. Sakarya Matbaacılık. Trabzon.

[19] Göktürk T., Artvinli T., Bucak F. 2008. Artvin Kuş Faunası. Artvin Çoruh Üniversitesi Orman Fakültesi Dergisi, 9 (1-2): 33-43. 